\title{
CARACTERIZAÇÃO COMPLETA DA MADEIRA DA ESPÉCIE AMAZÔNICA PARICÁ (Schizolobium amazonicum HERB) EM PEÇAS DE DIMENSÕES ESTRUTURAIS ${ }^{1}$
}

\author{
Diego Henrique de Almeida ${ }^{2}$, Ricardo de Mello Scaliante ${ }^{3}$, Laurenn Borges de Macedo², Alcebíades
} Negrão Macêdo ${ }^{4}$, Antonio Alves Dias ${ }^{5}$, André Luis Christoforo ${ }^{6}$ e Carlito Calil Junior ${ }^{5}$

\begin{abstract}
RESUMO - A espécie amazônica Paricá (Schizolobium amazonicum Herb) apresenta rápidos incrementos em altura e diâmetro em poucos anos de cultivo e desenvolve uma madeira com pequena quantidade de nós e defeitos, característica fundamental na sua utilização para fins estruturais. O objetivo deste trabalho foi determinar as propriedades de resistência e rigidez de peças da madeira Paricá em dimensões estruturais, de acordo com a norma ABNT NBR 7190: 2011, bem como realizar a classificação visual das peças para determinação de defeitos como: nós, inclinação de fibras, empenamentos, rachas e fendas. Conclui-se que a madeira de Paricá é classificada como classe C 20 das dicotiledôneas e possui baixa densidade, boa resistência à compressão paralela, grande potencial de utilização na construção civil quando comparada com outras espécies e, em relação à classificação visual, poucos nós, grã direita e poucos empenamentos e distorções.
\end{abstract}

Palavras-chave: Caracterização completa; Estruturas de madeira; Madeira.

\section{STRUCTURAL CHARACTERIZATION OF THE AMAZONIAN WOOD SPECIE PARICÁ (Schizolobium amazonicum HERB) IN MEMBERS}

\begin{abstract}
The Amazon specie Paricá (Schizolobium amazonicum Herb) present fasts growth and very good development in diameter in a few years, a small amount of nodes and defects, key characteristic in its use for structural purpose. The aim of this paper was to determine the strength and stiffness properties of Paricá wood, according to ABNT NBR 7190: 2011, as well, perform the visual classification of members for determination of defects as: nodes, inclination of grain, warpings. The concluding that the Paricá wood is classified as C 20 dicotyledonous class, low density, with good compression strength parallel to the grain, with a great potential to be used in civil construction, when compared with other species and, in relation to the visual analysis, few nodes, right grain and has no warping.
\end{abstract}

Keywords: Full characterization; Timber structures; Wood.

\footnotetext{
${ }^{1}$ Recebido em 14.02.2013 aceito para publicação em 15.10.2013.

${ }^{2}$ Laboratório de Madeiras e de Estruturas de Madeira, Departamento de Engenharia de Estruturas, Escola de Engenharia de São Carlos, Universidade de São Paulo, USP. E-mail: <almeidadh@usp.br> e <laurennmacedo@hotmail.com>.

${ }^{3}$ Departamento Nacional de Infraestrutura de Transportes, DNIT. E-mail:<ricscaliante@yahoo.com.br>.

${ }^{4}$ Universidade Federal do Pará, Instituto de Tecnologia. E-mail: <anmacedo@ufpa.br>.

${ }^{5}$ Departamento de Engenharia de Estruturas, Escola de Engenharia de São Carlos, Universidade de São Paulo, USP. E-mail: <dias@sc.usp.br>e <calil@sc.usp.br>.

${ }^{6}$ Departamento de Engenharia Mecânica, Universidade Federal de São João del Rei, UFSJ. E-mail: <alchristoforo@yahoo.com.br>.
}

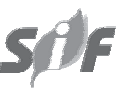

Revista Árvore, Viçosa-MG, v.37, n.6, p.1175-1181, 2013 


\section{INTRODUÇÃO}

A partir do cultivo de florestas, inúmeros subprodutos podem ser utilizados pelo homem para suprir suas necessidades. Um desses subprodutos que há muito tempo é utilizado pela humanidade é a madeira serrada, que teve suas primeiras atribuições com o intuito de oferecer subsídios para a construção, mesmo que rústica, de edificações para proteção e moradia e para ultrapassar obstáculos impostos pela natureza.

Com o passar dos tempos, o homem passou a explorar outros materiais que substituíam a madeira, porém, atualmente, a demanda de produtos e serviços que prejudiquem o mínimo possível o meio ambiente está dominando o cenário mundial. Nesse tocante, a madeira serrada proveniente de florestas plantadas se torna material com potencial para diversos setores da economia.

No Brasil existem florestas plantadas de espécies de árvores exóticas como as dos gêneros Eucalyptus, Pinus e Corymbia, que são os principais utilizados em reflorestamentos. Sem contar que a região amazônica brasileira apresenta imenso contingente de espécies de madeiras ainda inexploradas. O Paricá (Schizolobium amazonicum Herb), espécie amazônica de grande potencial econômico, tem conquistado cada vez mais destaque entre as espécies reflorestadas no Brasil, pois possui incrementos em altura e diâmetro que possibilitam sua utilização em poucos anos.

De acordo com Marques et al. (2006), a madeira de Paricá apresenta facilidade em relação à remoção da casca, laminação, secagem, prensagem e excelente acabamento. Segundo Carvalho (2007), em algumas cidades do interior do Pará, reflorestamentos em torno de 6 anos de idade atingiram produção volumétrica de $38 \mathrm{~m}^{3} / \mathrm{ha}$ /ano.

A utilização de madeira proveniente de florestas plantadas e devidamente manejadas fornece matériaprima para diversos ramos da indústria e de prestação de serviços no Brasil. Um dos principais meios de utilização da madeira é a construção civil, que a utiliza como elemento estrutural.

Para utilização da madeira como elemento estrutural, algumas precauções devem ser tomadas para que a estrutura seja bem construída e resista às solicitações impostas durante a sua utilização.
Antes de a madeira ser empregada numa construção civil, seja ela uma casa, telhado, ponte, silo ou qualquer outra utilização de peças de madeira que se comportem como elemento estrutural, é imprescindível a classificação e caracterização das suas propriedades físicas e mecânicas, que são as principais propriedades da madeira a serem levadas em conta quando utilizada em estruturas.

As propriedades mecânicas da madeira são divididas em propriedades de resistência e em propriedades de rigidez. Entre as propriedades de resistência, podem-se citar a resistência à compressão paralela às fibras e o módulo de resistência à flexão na direção paralela às fibras. Já em relação às propriedades de rigidez podemos citar os módulos de elasticidade longitudinal e transversal.

Uma das principais características da madeira, e que interfere na sua utilização, é a característica higroscópica, ou seja, facilidades a mudanças do teor de umidade em função do local onde está empregada (GALVÃO; JANKOWSKY, 1985). O Ponto de Saturação das Fibras (PSF) possui valor variável, dependendo da espécie de madeira utilizada, variando de $22 \%$ para coníferas com alto teor de resina até 35\% para folhosas com grande porosidade no alburno. Abaixo do PSF ocorrem alterações significativas nas propriedades físicomecânicas da madeira (LOGSDON; CALIL JUNIOR, 2002).

A Associação Brasileira de Normas Técnicas (ABNT), em seu documento número 7190, de 1997, denominado "Projeto de Estruturas de Madeira” (ABNT NBR 7190:1997), fornece em seu Anexo B “Determinação das propriedades das madeiras para projetos de estruturas" as diretrizes para realização dos ensaios laboratoriais para determinação das propriedades físicomecânicas da madeira, utilizando corpos de prova livres de defeitos em dimensões padronizadas.

O texto-base redigido para a nova versão do documento 7190 da ABNT (ABNT NBR 7190: 2011) contempla ensaios laboratoriais em peças de madeira com dimensões estruturais e também a classificação visual dessas peças.

A classificação visual consiste na inspeção não destrutiva das faces, lados (bordas laterais) e extremidades de cada peça. Devem-se examinar todo o comprimento das peças e avaliar a localização e natureza dos nós e outros defeitos presentes na sua superfície (CALIL NETO, 2011; CARREIRA, 2003).

Revista Árvore, Viçosa-MG, v.37, n.6, p.1175-1181, 2013 
A partir de características das peças que podem ser observadas visualmente - entre elas inclinação das fibras, nós, rachas, fendas e empenamentos -, são definidas quatro classes, de acordo com a presença de defeitos: Classe Estrutural Especial (SE), Classe Estrutural $n^{\circ} 1$ (S1), Classe Estrutural $n^{\circ} 2$ (S2) e Classe Estrutural no 3 (S3).

Após a classificação visual, é necessário realizar a classificação mecânica da madeira por intermédio de ensaios laboratoriais, para determinação de suas propriedades de resistência e rigidez.

O objetivo deste trabalho foi determinar as propriedades de resistência e rigidez em peças de dimensões estruturais da madeira de Paricá, bem como a classificação a partir de características visuais dessas peças de acordo com as diretrizes fornecidas pela norma brasileira de madeiras.

\section{MATERIAL E MÉTODOS}

Neste estudo foram utilizadas peças de madeira de dimensões estruturais da madeira da espécie Paricá (Schizolobium amazonicum Herb), proveniente do Estado do Pará, com 10 anos de idade. A partir de um lote homogêneo, foram realizadas as classificações visual e mecânica em peças com as seguintes dimensões: $230 \mathrm{~cm}$ de comprimento, $15 \mathrm{~cm}$ de largura e 3,5 cm de espessura. Após, foram realizados os seguintes ensaios de caracterização, de acordo com a norma brasileira ABNT NBR 7190: 2011 (Figura 1):

- Resistência à compressão paralela às fibras ( $\left.\mathrm{f}_{\mathrm{c} 0}\right)$.

- Módulo de elasticidade na compressão paralela às fibras $\left(\mathrm{E}_{\mathrm{c} 0}\right)$.

- Módulo de elasticidade na flexão (MOE).

- Módulo de ruptura na flexão (MOR).

- Resistência ao cisalhamento paralelo às fibras $\left(\mathrm{f}_{\mathrm{v} 0}\right)$.

- Resistência à tração paralela às fibras $\left(\mathrm{f}_{\mathrm{t} 0}\right)$.

- Densidade básica $\left(\rho_{\text {bas }}\right)$.

- Densidade aparente $\left(\rho_{\text {ap }}\right)$.

- Teor de umidade (TU).

Os ensaios foram realizados no Laboratório de Madeiras e de Estruturas de Madeira (LaMEM), do Departamento de Estruturas (SET) da Escola de Engenharia de São Carlos (EESC), da Universidade de São Paulo (USP).
De acordo com a norma brasileira, foram determinados os valores característicos de resistência utilizando a equação a seguir:

$$
f_{k}=\left(2 \cdot \frac{f_{1}+f_{2}+\ldots \frac{f_{n}}{2}-1}{\frac{n}{2}-1}-\frac{f_{n}}{2}\right) \cdot 1,1
$$

em que $\mathrm{f}_{\mathrm{k}}$ é o valor característico de resistência, n é o número de corpos de prova utilizados, e os respectivos resultados de resistência devem ser colocados em ordem crescente $\mathrm{f}_{1} \leq \mathrm{f}_{2} \leq \ldots \leq \mathrm{f}_{\mathrm{n}}$, desprezando o valor mais alto se o número de corpos de prova for ímpar, não se tomando para $\mathrm{f}_{\mathrm{k}}$ valor inferior a $\mathrm{f}_{1}$, nem a $70 \%$ do valor médio.

Os valores médios de módulo de elasticidade a partir dos ensaios de compressão paralela em relação às fibras e de flexão foram determinados para caracterização completa da rigidez da madeira.

Para corrigir os valores de resistência obtidos em ensaio para o teor de umidade de referência (12\%), a atual norma brasileira adota a equação a seguir para teores de umidade entre $10 \%$ e $20 \%$. Os procedimentos para determinação do teor de umidade da madeira estão contidos no Anexo B da referida norma.

$$
\mathrm{f}_{12}=\mathrm{f}_{\mathrm{u} \%} \cdot\left(1+\frac{3 \cdot(\mathrm{U} \%-12)}{100}\right)
$$

em que $\mathrm{f}_{12}$ é a resistência corrigida ao teor de umidade de $12 \%, \mathrm{f}_{\mathrm{u} \%}$ é a resistência determinada no teor de umidade da madeira no instante do ensaio e U\% é o teor de umidade da madeira no ambiente.

\section{RESULTADOS}

As densidades básica e aparente determinadas para a madeira de Paricá foram de $0,30 \mathrm{~g} / \mathrm{cm}^{3}$ e 0,37 $\mathrm{g} / \mathrm{cm}^{3}$, respectivamente, utilizando-se 30 corpos de prova. Bianche et al. (2012) e Macedo et al. (2012) determinaram para o Paricá densidades básicas iguais a $0,26 \mathrm{~g} / \mathrm{cm}^{3}$ e $0,28 \mathrm{~g} / \mathrm{cm}^{3}$, respectivamente, mostrando como a madeira é material heterogêneo com as propriedades diferentes até mesmo em indivíduos de mesma espécie. De acordo com Loureiro et al. (1997), algumas essências amazônicas apresentam a densidade básica semelhante à do Paricá, como o caso da Caroba (Jacaranda copaia D. Don), da Castanha-de-arara (Joannesia heveoides Ducke) e do Urucum-da-mata 
(Bixa arborea Huber). Foi constatado nas madeiras utilizadas nos ensaios desta caracterização um teor de umidade igual a $13 \%$.

A Tabela 1 apresenta os valores médios e característicos de resistência para caracterização completa em corpos de prova de dimensões estruturais da espécie Paricá, os valores médios de rigidez, os desvios-padrão, os coeficientes de variação (C.V.) e, também, a quantidade de corpos de prova utilizados em cada ensaio. Todos esses resultados foram corrigidos para o teor de umidade de $12 \%$, de acordo com a norma ABNT NBR 7190: 2011.

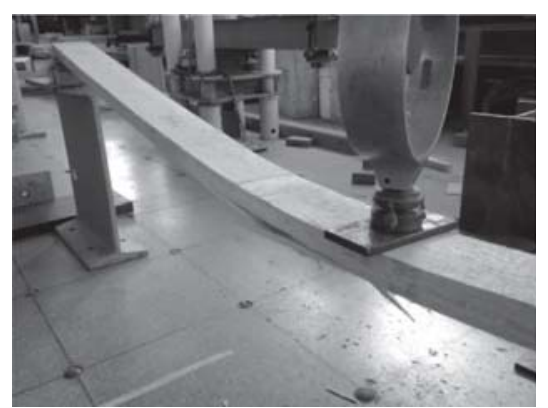

(a)

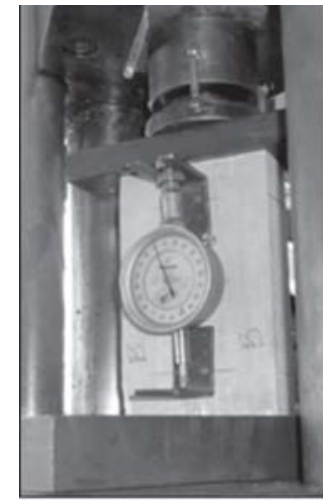

(b)

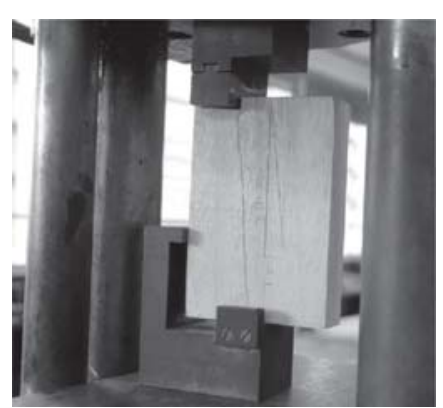

(c)

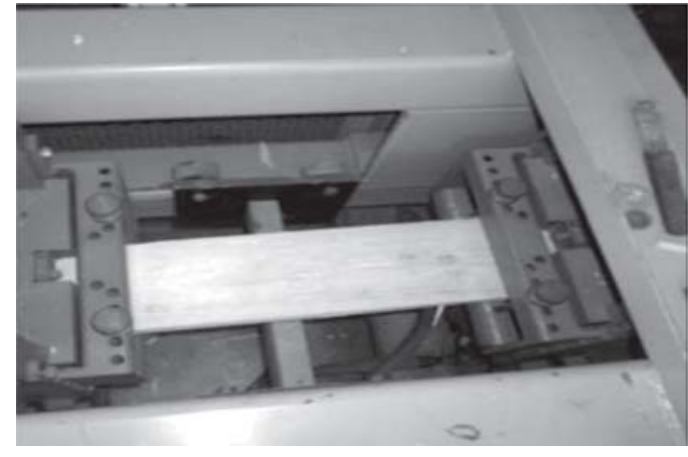

(d)

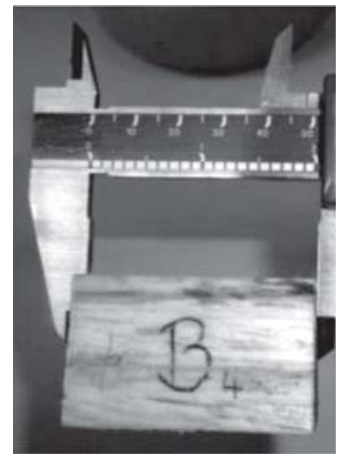

(e)

Figura 1 - Corpos de prova instrumentados para ensaios mecânicos de (a) flexão estática, (b) compressão estrutural, (c) cisalhamento estrutural e (d) tração estrutural paralelo às fibras e (e) ensaios físicos de densidade básica, densidade aparente e teor de umidade.

Figure 1 - Instrumented specimens for mechanical tests for (a) bending, (b) structural compression, (c) structural shear and (d) structural tension parallel to grain and (e) physics tests of basic density, apparent density and moisture content.

Tabela 1 - Valores médios de resistência e rigidez, desvio-padrão, coeficiente de variação, valores característicos de resistência e número de corpos de prova.

Table 1 - Average values of strength and stiffness, standard deviation, variation coefficient, characteristic values of strength and number of specimens.

\begin{tabular}{lcccccc}
\hline Propriedades & $\mathrm{f}_{\mathrm{c} 0}(\mathrm{MPa})$ & $\mathrm{E}_{\mathrm{c} 0}(\mathrm{MPa})$ & $\mathrm{f}_{\mathrm{t} 0}(\mathrm{MPa})$ & $\mathrm{f}_{\mathrm{v} 0}(\mathrm{MPa})$ & $\mathrm{MOE}(\mathrm{MPa})$ & $\mathrm{MOR}(\mathrm{MPa})$ \\
\hline $\mathrm{n}^{\circ}$ corpos de prova & 15 & 15 & 6 & 6 & 12 & 12 \\
Valores médios & 24 & 7320 & 40 & 6 & 8900 & 50 \\
Desvios-padrão & 4 & 1280 & 16 & 1,30 & 1800 & 14 \\
C.V. (\%) & 17 & 18 & 40 & 22 & 20 & 27 \\
Valores característicos & 21 & - & 25 & 4 & - & 32 \\
\hline
\end{tabular}

Revista Árvore, Viçosa-MG, v.37, n.6, p.1175-1181, 2013 


\section{DISCUSSÕES}

O valor de $\mathrm{f}_{\mathrm{c} 0, \mathrm{k}}$ igual a $21 \mathrm{MPa}$, de acordo com a norma brasileira, classifica a espécie Paricá como madeira dicotiledônea de classe de resistência C 20, valor esse próximo ao determinado por Vidaurre et al. (2012).

A ruptura do corpo de prova na flexão estática se caracteriza pela tração na direção paralela em relação às fibras. O módulo de ruptura na flexão possui valor inferior à resistência característica à tração paralela às fibras em corpo de prova em dimensões estruturais da ordem de $28 \%$.

Almeida et al. (2011) estudaram vigas de Madeira Laminada Colada (MLC) com madeira de Paricá utilizando lamelas classificadas visualmente como Classe Estrutural
Especial (SE), ressaltando sobre a qualidade da madeira dessa espécie na confecção de MLC, pois ela possui boa aderência com o adesivo utilizado e, por ser madeira de baixa densidade, proporciona vigas de alta relação resistência-peso específico.

A classificação visual realizada nas peças mostrou que a madeira de Paricá não apresentou muitos nós, sendo os poucos existentes muito pequenos, não causando prejuízo expressivo à seção transversal da peça. A inclinação das fibras nas peças estudadas também é desprezível, possuindo grã reta. As peças estudadas também não possuíam rachas e fendas, tampouco algum tipo de empenamento. A partir da classificação visual das peças estruturais da madeira de Paricá, pode-se concluir que ela é classificada como Classe Estrutural Especial (SE).

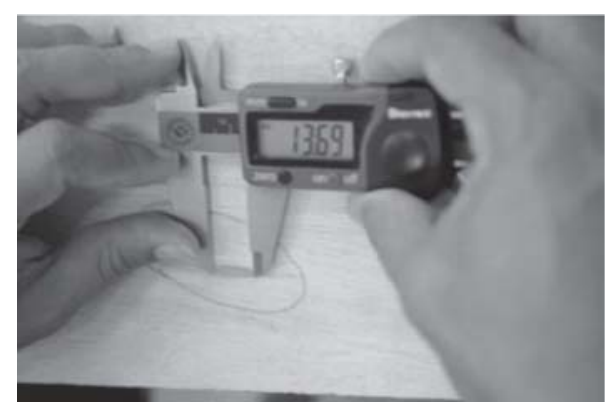

(a)

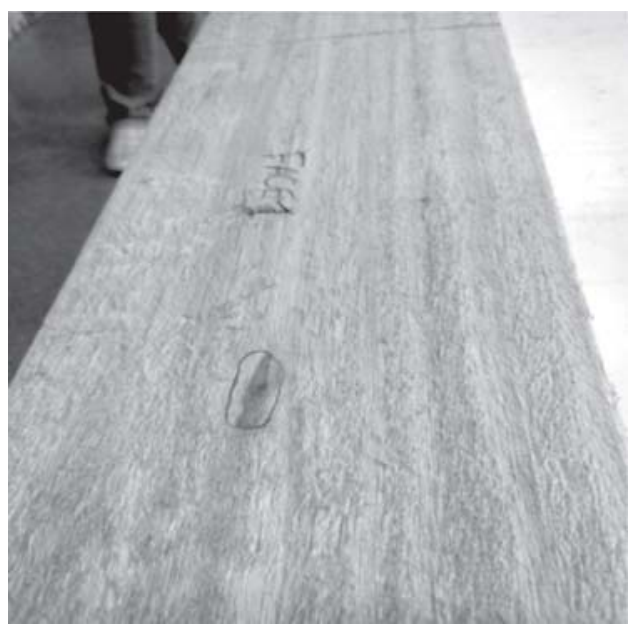

(c)

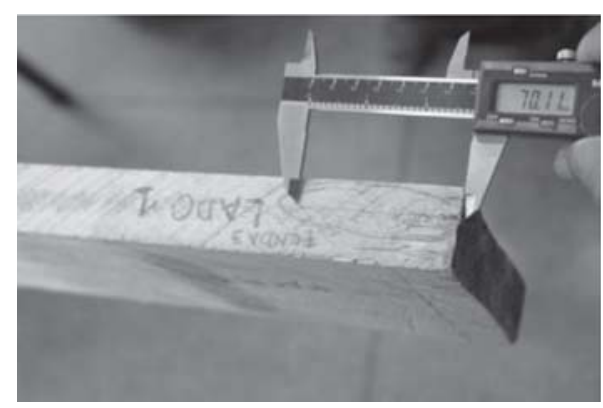

(b)

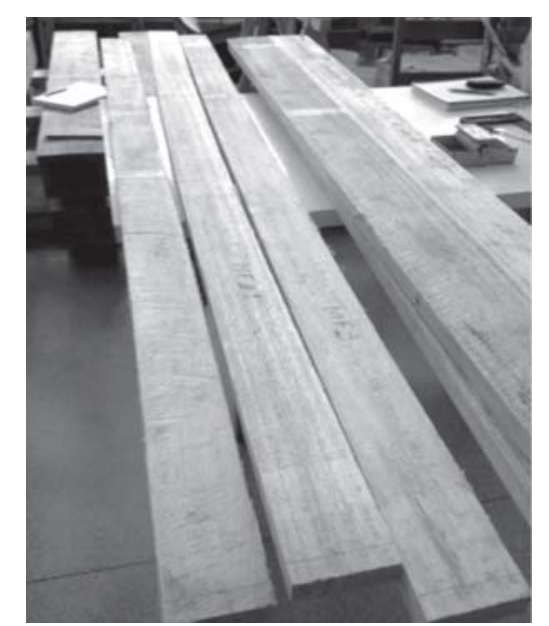

(d)

Figura 2 - Peças de madeira estruturais analisadas visualmente: (a) diâmetro dos nós, (b) comprimento de rachas e fendas, (c) inclinação das fibras e (d) empenamentos.

Figure 2 - Structural timber member visually analisated: (a) diameter nodes, (b) cracks and crevices length, (c) inclination of grain and (d) warpings. 
Comparando a madeira de Paricá classificada visualmente com a madeira das espécies Pinus elliottii (Pinus elliottii var. elliottii) e Pinus taeda (Pinus taeda L.), observou-se que a espécie amazônica possui menor incidência de nós (PUCCINI et al., 2002; CARREIRA; DIAS, 2005; ECKERT, 2011).

Determinaram-se os valores para relação $f_{c 0} / \rho_{a p}$, nas madeiras de Paricá, Pinus elliottii e Pinus taeda iguais a 73, 72 e 69, respectivamente, utilizando os dados encontrados neste trabalho e na norma ABNT NBR 7190: 1997. A madeira da espécie Paricá apresenta resistência à compressão paralela superior às determinadas para madeiras do gênero Pinus, mesmo possuindo densidade inferior.

\section{CONCLUSÕES}

Dos ensaios realizados nas peças estruturais, pode-se concluir que a madeira de Paricá é classificada, em relação às suas propriedades de resistência e rigidez, como madeira C 20 da classe das dicotiledôneas. A classificação visual permitiu concluir que a madeira proveniente dessa espécie amazônica possui pequeno índice de defeitos, pequena quantidade de nós, grã direita e, também, poucos empenamentos. Relacionando a resistência à compressão paralela às fibras com a densidade aparente da madeira, o Paricá possui quociente superior ou igual a outras madeiras utilizadas na construção civil, a exemplo das do gênero Pinus.

\section{AGRADECIMENTOS}

Pelo apoio financeiro, os autores agradecem à Coordenação de Aperfeiçoamento de Pessoal de Nível Superior (CAPES) e ao Conselho Nacional de Desenvolvimento Científico e Tecnológico (CNPq).

\section{REFERÊNCIAS}

ALMEIDA, D. H. et al. Madeira laminada colada (MLC) da espécie Paricá. Madeira: Arquitetura e Engenharia, v.12, n.30, p.71-82, 2011.

ASSOCIAÇÃO BRASILEIRA DE NORMAS TÉCNICAS - ABNT. NBR 7190: Projetos de estruturas de madeiras. Rio de Janeiro: 2011 (Aprovada).
ASSOCIAÇÃO BRASILEIRA DE NORMAS TÉCNICAS - ABNT. NBR 7190: Projetos de estruturas de madeira. Rio de Janeiro: 1997.

BIANCHE, J. J. et al. Caracterização física e química das madeiras de Paricá, Eucalipto e caule de Vassoura visando a produção de painéis. In: ENCONTRO BRASILEIRO EM MADEIRAS E EM ESTRUTURAS DE MADEIRA - EBRAMEM, 13., 2012, Vitória. Anais... Vitória: UFES, 2012. CD ROM.

CALIL NETO, C. Madeira laminada colada (MLC): controle de qualidade em combinações espécie - adesivo - tratamento preservativo. 2011. 120f. Dissertação (Mestrado em Engenharia e Ciências dos Materiais) - Escola de Engenharia de São Carlos, Universidade de São Paulo, São Carlos, 2011.

CARREIRA, M. R. Critérios para classificação visual de peças estruturais de Pinus sp. 2003. 182f. Dissertação (Mestrado em Engenharia de Estruturas) - Escola de Engenharia de São Carlos, Universidade de São Paulo, São Carlos, 2003.

CARREIRA, M. R.; DIAS, A. A. Classificação visual de coníferas: análise da aplicação do método norte-americano às espécies de Pinus sp plantadas no Brasil. Scientia Forestalis, n. 67, p.78-87, 2005.

CARVALHO, P. E. R. Paricá: Schizolobium amazonicum. Colombo: Embrapa, 2007. 8p.

ECKERT, J. A. Classificação de peças estruturais de madeira para vigas laminadas coladas. 2011. 52f. Monografia (Graduação em Engenharia Civil) - Faculdade Dinâmica das Cataratas, Foz do Iguaçu, 2011.

GALVÃO, A. P. M.; JANKOWSKY, I. P.

Secagem racional da madeira. São Paulo: Nobel, 1985. 111p.

LOGSDON, N. B.; CALIL JUNIOR, C. Influência da umidade nas propriedades de resistência e rigidez da madeira, Caderno de Engenharia de Estruturas, n.18, p.77-107, 2002.

LOUREIRO, A. A.; FREITAS, J. A.; FREITAS, C. A. A. Essências madeireiras da Amazônia. Manaus: MCT/INPA-CPPF, 1997. 103p. 
MACEDO, L. B. et al. Caracterização de algumas propriedades físicas da madeira de Paricá (Schizolobium amazonicum Herb). In: CONGRESSO NACIONAL DE ENGENHARIA MECÂNICA, METALÚRGICA E INDUSTRIAL CONEMI, 12., 2012, São João del Rei. Anais... São João del Rei: UFSJ, 2012. CD ROM.

MARQUES, L. C. T.; YARED, J. A. G.; SIVIERO, M. A. A evolução do conhecimento sobre Paricá para o reflorestamento no estado do Pará. Belém: Embrapa Pará, 2006. 5p.
PUCCINI, C. T.; GONÇALVES, R.; MONTEIRO, M. E. A. Avaliação estatística da variação da velocidade de propagação de ondas de ultra-som na madeira em presença de defeitos. Revista Brasileira de Engenharia Agrícola e Ambiental, v.6, n.3, p.499-503, 2002.

VIDAURRE, G. et al. Influência da idade na resistência à flexão e compressão paralela da madeira de Paricá. In: ENCONTRO BRASILEIRO EM MADEIRAS E EM ESTRUTURAS DE MADEIRA - EBRAMEM, 13., 2012, Vitória. Anais... Vitória: UFES, 2012. CD ROM. 
